\title{
Energy Harvesting Two-Way Half-Duplex Relay Channel with Decode-and-Forward Relaying: Optimum Power Policies
}

\author{
Kaya Tutuncuoglu, Burak Varan and Aylin Yener \\ Electrical Engineering Department \\ The Pennsylvania State University, University Park, PA 16802 \\ kaya@psu.edu varan@psu.edu yener@ee.psu.edu
}

\begin{abstract}
In this paper, a half-duplex two-way relay channel with energy harvesting nodes is considered. In particular, shortterm throughput maximization problems are solved using a decode-and-forward relay. Necessary properties of the optimal transmission policy are derived to gain insights into the optimal solution. Then, a subgradient descent algorithm is used to find the optimal policy. It is observed through simulations that energydeficient nodes act as bottlenecks on the achieved throughput. The achieved average throughput is observed to be close to the upper bound when nodes have no energy intermittency, and significantly higher than the throughput achieved by naïve policies.
\end{abstract}

\section{INTRODUCTION}

We consider a bidirectional relay channel with energy harvesting nodes. Efficient energy management is required when dealing with such nodes since they harvest energy out of external sources in an intermittent fashion.

Recently, there has been considerable interest in energy harvesting networks [1]-[13]. A single-user wireless communication system is considered in [1] with random energy and data arrivals at the source. A piece-wise constant transmit power policy where the node transmits with constant power in intervals longest in duration while staying energy feasible, is shown to be optimal. Reference [2] considers the throughput maximization problem when the amount of energy that the battery can store is limited. The two problems in [1], [2] are observed to be related, and the optimal policy is shown to follow the shortest path within the feasible energy tunnel [2]. In [3], the extension of this model to fading channels is addressed. A single link scenario with an energy harvesting transmitter and receiver is analyzed in [4]. Multiple access, broadcast and interference channels with energy harvesting transmitters are considered in [5]-[9]. In addition to these multi-user setups, special cases of the energy harvesting relay channel problem have been studied in [10], [11], where it is noted that the problem becomes more challenging with a halfduplex relay and a data buffer at the relay.

In this work, we consider the two-way relay channel with energy harvesting nodes. The channel is two-hop in the sense that the transmitters cannot hear each other directly, i.e., communication is possible only through the relay. We consider a decode-and-forward relay [14], [15] operating in half-duplex mode. The choice of the relaying scheme is due to its relatively good performance, and its amenability to practical codes, as compared to, for instance, compress-and-forward. We formulate the short-term sum-rate maximization problem and identify properties of the optimal policy to gain insights into how the energy arrivals to the nodes affect the optimal policy and the resulting throughput. We observe that for nodes that are energy deprived, certain rate constraints have to be satisfied with equality, pointing to insights about efficient use of available energy. We then employ a subgradient descent method [16] to find the optimal solution to the problem. We observe through simulations that the optimal solution is dominated by the more energy-deficient group of nodes, i.e., transmitters or the relay, and thus the optimal solution is not necessarily unique contrary to previously studied energy harvesting setups [1], [2]. Comparing the rates achieved by the optimal policy with naïve policies, we confirm that the optimal policy performs notably better, approaching the upper bound where all harvested energy is available to the node at the beginning of transmission.

\section{SySTEM MODEL}

We consider an additive white Gaussian noise (AWGN) two-way relay channel with transmitters $T_{1}$ and $T_{2}$. These two transmitters wish to convey independent messages to each other, but cannot hear each other directly. They instead communicate via the relay node $T_{3}$. The two links in the channel are assumed to be reciprocal for simplicity, i.e., the link from $T_{1}$ (resp. $T_{2}$ ) to $T_{3}$ and the link from $T_{3}$ to $T_{1}$ (resp. $T_{2}$ ) have the same static fading coefficient $h_{13}$ (resp. $h_{23}$ ). We note that the results of the paper can easily be extended to models without reciprocal channel gains. The relay does not employ a data buffer, therefore it has to forward messages as soon as it receives them. The channel model is given in Figure 1. All channel coefficients and transmit powers are normalized with respect to the corresponding receiver noise so that the Gaussian noise of each link has an effective variance of 1 . This is achieved by first normalizing $h_{1,3}$ and $h_{2,3}$ to establish unit variance noise at $T_{1}$ and $T_{2}$, and subsequently 


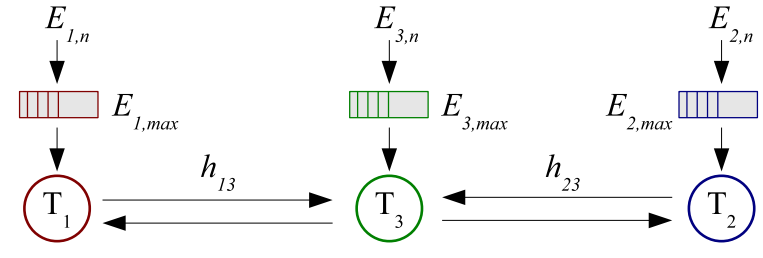

Fig. 1: The separated two-way relay channel with energy harvesting nodes.

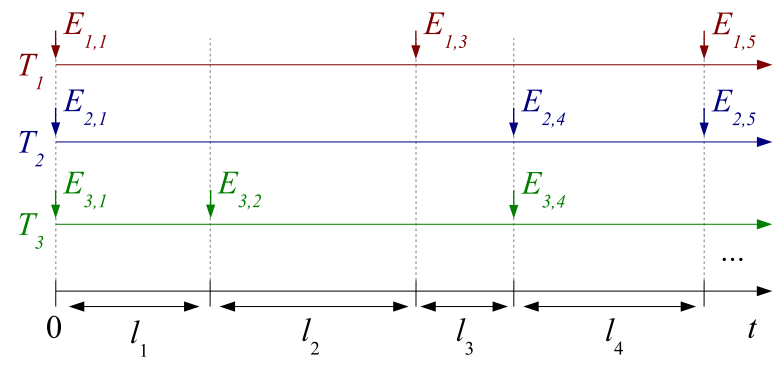

Fig. 2: The energy harvesting model for node $j$. Instances with $E_{j, n}=0$ are not shown.

scaling the transmit power at nodes $T_{1}$ and $T_{2}$ to do the same for the noise at $T_{3}$.

As shown in Figure 1, nodes do not have a constant source of energy; they are powered by harvested energy only. An energy packet of $E_{j, n}$ units arrives at node $j$ at the $n^{\text {th }}$ energy arrival for all $j=1,2,3$. Harvested energy is stored in a battery with finite capacity $E_{j, \max }$ for node $j$, so any energy in excess of the capacity of the battery is lost. We refer to the time duration between the $n^{t h}$ and $(n+1)^{s t}$ energy arrivals as the $n^{t h}$ epoch and the length of the $n^{t h}$ epoch is denoted by $l_{n}$. The epochs are constructed by the union of all arrivals to all nodes, similar to references [5], [10], but the nodes do not necessarily harvest energy all at the same time. Therefore, zero energy arrivals are allowed and $E_{j, n}$ is set to 0 if node $j$ does not have an energy arrival at the beginning of the $n^{\text {th }}$ epoch. $E_{j, 1}$ denotes the initial amount of energy in the battery of node $j$ just before transmission starts. Figure 2 depicts an example of the construction of epochs in our energy harvesting model.

The energy harvesting profiles described by $E_{j, n}$ and $l_{n}$ play an important role on the feasibility of a transmission policy as the energy available to each node is strictly limited to the energy stored in its battery. Specifically, the energy required by each node for a feasible transmission policy should not exceed the energy available, both considering the amount of harvested energy and the capacity of energy storage device. In this work, it is assumed that all energy harvests are known non-causally by the transmitters and the relay, so that offline optimal policies and performance limits of the network can be found.

We denote the power policy of the network by vectors $\mathbf{p}_{1}$, $\mathbf{p}_{2}$ and $\mathbf{p}_{3}$, where each vector represents the average power allocated to the respective node in each epoch. In order to formulate a set for feasible power policies, we first present the following observation:

Observation 1: There exists an optimal power policy that never allows any of the batteries to overflow.

The proof follows from the fact that given any power policy with battery overflows, the node can withdraw more power from its battery prior to an overflow, potentially enlarging the set of rates it can achieve, while preventing the overflow and keeping battery state the same after the overflow. This is possible due to the non-causal knowledge of harvested energy packets, and was shown in detail in previous work [2]. Thus, restricting the feasible set of policies to one that does not overflow the battery is guaranteed to admit an optimal policy that is also optimal when battery overflows are allowed.

With the no-overflow restriction, the set of feasible policies are defined by two sets of constraints,

$$
\begin{aligned}
& \sum_{i=1}^{n} l_{i} p_{j, i}-\sum_{i=1}^{n} E_{j, i} \leq 0 \\
& \sum_{i=1}^{n} E_{j, i}-\sum_{i=1}^{n-1} l_{i} p_{j, i} \leq E_{j, \max }
\end{aligned}
$$

for all $j=1,2,3$ and $n=1,2, \ldots, N$ where $N$ is the number of epochs considered in the problem. Energy causality constraints, given in (1), ensure that the energy consumed by a node does not exceed the energy harvested up to that epoch. No-overflow constraints, given in (2), imply that whenever the $n+1^{\text {st }}$ energy packet arrives to a node, the battery can store all of this energy without any overflows. Any policy $\left(\mathbf{p}_{1}, \mathbf{p}_{2}, \mathbf{p}_{3}\right)$ satisfying both (1) and (2) for all $j$ and $i$ is considered a feasible policy.

The communication model is the so-called multiple-access broadcast (MABC) decode-and-forward [17]. The MABC decode-and-forward scheme consists of a multiple-access channel from transmitters $T_{1}$ and $T_{2}$ to $T_{3}$ and a broadcast channel from $T_{3}$ to $T_{1}$ and $T_{2}$, which will be referred to as the multiple-access and broadcast phases in the sequel. The two phases occur sequentially in each epoch. The fraction of the length of the epoch $l_{i}$ allocated to the broadcast phase is denoted by the time-sharing parameter $0 \leq \Delta \leq 1$. This is another parameter of the transmission policy that needs to be optimized. Reference [15] shows the instantaneous rates achieved by MABC in a half-duplex setup to be

$$
\begin{aligned}
& R_{1} \leq \min \left\{\Delta C\left(\frac{\left|h_{13}\right|^{2} p_{1}}{\Delta}\right),(1-\Delta) C\left(\frac{\left|h_{23}\right|^{2} p_{3}}{1-\Delta}\right)\right\} \\
& R_{2} \leq \min \left\{\Delta C\left(\frac{\left|h_{23}\right|^{2} p_{2}}{\Delta}\right),(1-\Delta) C\left(\frac{\left|h_{13}\right|^{2} p_{3}}{1-\Delta}\right)\right\} \\
& R_{1}+R_{2} \leq \Delta C\left(\frac{\left|h_{13}\right|^{2} p_{1}}{\Delta}+\frac{\left|h_{23}\right|^{2} p_{2}}{\Delta}\right)
\end{aligned}
$$

where $R_{1}$ and $R_{2}$ are the rates of the messages originating from nodes $T_{1}$ and $T_{2}$ respectively, $p_{1}, p_{2}$ and $p_{3}$ are the 
average powers consumed by nodes $T_{1}, T_{2}$ and $T_{3}$ respectively and $C(p)=\frac{1}{2} \log (1+p)$. The instantaneous transmit powers in each phase is found by scaling the average consumed power with $\Delta$. Note that in the broadcast phase, the relay transmits a function of the two decoded messages, which can be decoded at $T_{1}$ and $T_{2}$ using their own messages to find the intended ones. Therefore, in the broadcast phase, $T_{1}$ and $T_{2}$ only need to decode at the rate they intend to receive the message from the other node. The multiple access and broadcast phases are depicted in Figure 1.

\section{Optimal Power Allocation}

We address the problem of maximizing the throughput for the half-duplex two-way relay channel by choosing the average powers $p_{j}$ in each epoch $i$ for each node $j=1,2,3$. In what follows, we will state the problem and use KKT analysis to extract properties of the optimal solution.

For a power policy to be feasible, each node must satisfy its energy causality and battery capacity constraints, and the resulting instantaneous rates must be drawn from the region defined in (3). Then, the sum-rate maximization problem is formulated as

$$
\begin{aligned}
\underset{\substack{\mathbf{R}_{1}, \mathbf{R}_{2}, \Delta \\
\mathbf{p}_{1}, \mathbf{p}_{2}, \mathbf{p}_{3}}}{\max } & \sum_{n=1}^{N} l_{n}\left(R_{1, n}+R_{2, n}\right) \\
\text { s.t. } & \sum_{i=1}^{n} l_{i} p_{j, i} \leq \sum_{i=1}^{n} E_{j, i}, \\
& \sum_{i=1}^{n} E_{j, i}-\sum_{i=1}^{n-1} l_{i} p_{j, i} \leq E_{j, \max } \\
& R_{k, n} \leq \Delta_{n} C\left(\frac{h_{k 3}^{2} p_{k, n}}{\Delta_{n}}\right), \\
& R_{1, n}+R_{2, n} \leq \Delta_{n} C\left(\frac{h_{13}^{2} p_{1, n}+h_{23}^{2} p_{2, n}}{\Delta_{n}}\right), \\
& R_{k, n} \leq\left(1-\Delta_{n}\right) C\left(\frac{h_{\bar{k} 3}^{2} p_{3, n}}{1-\Delta_{n}}\right), \\
& R_{k, n} \geq 0, \quad 0 \leq \Delta_{n} \leq 1, \quad j=1,2,3, \\
& k, \bar{k}=1,2, \quad \bar{k} \neq k, \quad n=1,2, \ldots, N
\end{aligned}
$$

where

$$
\begin{array}{rlrl}
\mathbf{R}_{k} & =\left(R_{k, 1}, R_{k, 2}, \ldots, R_{k, N}\right), & k=1,2, \\
\mathbf{p}_{j} & =\left(p_{j, 1}, p_{j, 2}, \ldots, p_{j, N}\right), & & j=1,2,3 . \\
\boldsymbol{\Delta} & =\left(\Delta_{1}, \Delta_{2}, \ldots, \Delta_{N}\right) & &
\end{array}
$$

Here, $R_{1, n}$ and $R_{2, n}$ denote the rates in the $n^{\text {th }}$ epoch for the messages originating from nodes $T_{1}$ and $T_{2}$ respectively, $\mathbf{p}_{1}, \mathbf{p}_{2}$ and $\mathbf{p}_{3}$ denote the transmission power vectors, and $\boldsymbol{\Delta}$ is the vector of time-sharing parameters. Constraints (4b) are the energy causality constraints and (4c) are the battery capacity constraints for $T_{1}, T_{2}$ and $T_{3}$. Constraints (4d) and (4e) are the rate restrictions due to the multiple-access phase and (4f) are the rate restrictions due to the broadcast phase and non-negativity. All the constraints in (4) are convex and the objective function is concave. Therefore, (4) is a convex optimization problem, and yields an optimal solution that satisfies the Karush-Kuhn-Tucker (KKT) conditions. Taking the partial derivatives of the Lagrangian of (4), the stationarity conditions are found as

$$
\begin{aligned}
& v_{1, n}+v_{2, n}+v_{5, n}-\mu_{1, n}=l_{n}, \\
& v_{3, n}+v_{4, n}+v_{5, n}-\mu_{2, n}=l_{n}, \\
& \left(\sum_{m=n}^{N} u_{1, m}-\sum_{m=n+1}^{N} w_{1, m}\right) l_{n}= \\
& \frac{1}{\ln 2}\left(\frac{v_{1, n} h_{13}^{2}}{1+\frac{h_{13}^{2} p_{1, n}}{\Delta_{n}}}+\frac{v_{5, n} h_{13}^{2}}{1+\frac{h_{13}^{2} p_{1, n}+h_{23}^{2} p_{2, n}}{\Delta_{n}}}\right) \\
& \left(\sum_{m=n}^{N} u_{2, m}-\sum_{m=n+1}^{N} w_{2, m}\right) l_{n}= \\
& \frac{1}{\ln 2}\left(\frac{v_{3, n} h_{23}^{2}}{1+\frac{h_{23}^{2} p_{2, n}}{\Delta_{n}}}+\frac{v_{5, n} h_{23}^{2}}{1+\frac{h_{13}^{2} p_{1, n}+h_{23}^{2} p_{2, n}}{\Delta_{n}}}\right) \\
& \left(\sum_{m=n}^{N} u_{3, m}-\sum_{m=n+1}^{N} w_{3, m}\right) l_{n}= \\
& \frac{1}{\ln 2}\left(\frac{v_{2, n} h_{23}^{2}}{1+\frac{h_{23}^{2} p_{3, n}}{1-\Delta_{n}}}+\frac{v_{4, n} h_{13}^{2}}{1+\frac{h_{13}^{2} p_{3, n}}{1-\Delta_{n}}}\right) \\
& v_{1, n}\left(\frac{h_{13}^{2} p_{1, n}}{\ln 2\left(\Delta_{n}+h_{13}^{2} p_{1, n}\right)}-C\left(\frac{h_{13}^{2} p_{1, n}}{\Delta_{n}}\right)\right) \\
& -v_{2, n}\left(\frac{h_{23}^{2} p_{3, n}}{\ln 2\left(1-\Delta_{n}+h_{23}^{2} p_{3, n}\right)}-C\left(\frac{h_{23}^{2} p_{3, n}}{1-\Delta_{n}}\right)\right) \\
& +v_{3, n}\left(\frac{h_{23}^{2} p_{2, n}}{\ln 2\left(\Delta_{n}+h_{23}^{2} p_{2, n}\right)}-C\left(\frac{h_{23}^{2} p_{2, n}}{\Delta_{n}}\right)\right) \\
& -v_{4, n}\left(\frac{h_{13}^{2} p_{3, n}}{\ln 2\left(1-\Delta_{n}+h_{13}^{2} p_{3, n}\right)}-C\left(\frac{h_{13}^{2} p_{3, n}}{1-\Delta_{n}}\right)\right) \\
& +v_{5, n}\left(\frac{h_{13}^{2} p_{1, n}+h_{23}^{2} p_{2, n}}{\ln 2\left(\Delta_{n}+h_{13}^{2} p_{1, n}+h_{23}^{2} p_{2, n}\right)}\right. \\
& \left.-C\left(\frac{h_{13}^{2} p_{1, n}+h_{23}^{2} p_{2, n}}{\Delta_{n}}\right)\right)=\lambda_{1, n}-\lambda_{1, n}
\end{aligned}
$$

for all $n=1,2, \ldots, N$, where $v_{k, n}, u_{j, n}, w_{j, n}, \lambda_{1, n}$ and $\lambda_{2, n}$ are the Lagrangian multipliers for rate constraints, energy causality, battery capacity and time-sharing parameter constraints at the end of (4d), respectively. The complementary slackness conditions are

$$
\begin{aligned}
& u_{j, n} \sum_{i=1}^{n}\left(l_{i} p_{j, i}-E_{j, i}\right)=0 \\
& w_{j, n}\left(\sum_{i=1}^{n} E_{j, i}-\sum_{i=1}^{n-1} l_{i} p_{j, i}-E_{j, \max }\right)=0, \\
& v_{1, n}\left(R_{1, n}-\Delta_{n} C\left(h_{13}^{2} p_{1, n} / \Delta_{n}\right)\right)=0 \\
& v_{2, n}\left(R_{1, n}-\left(1-\Delta_{n}\right) C\left(h_{23}^{2} p_{3, n} /\left(1-\Delta_{n}\right)\right)\right)=0, \\
& v_{3, n}\left(R_{2, n}-\Delta_{n} C\left(h_{23}^{2} p_{2, n} / \Delta_{n}\right)\right)=0
\end{aligned}
$$




$$
\begin{aligned}
& v_{4, n}\left(R_{2, n}-\left(1-\Delta_{n}\right) C\left(h_{13}^{2} p_{3, n} /\left(1-\Delta_{n}\right)\right)\right)=0, \\
& v_{5, n}\left(R_{1, n}+R_{2, n}-\Delta_{n} C\left(\frac{h_{13}^{2} p_{1, n}+h_{23}^{2} p_{2, n}}{\Delta_{n}}\right)\right)=0, \\
& \mu_{1, n} R_{1, n}=0, \quad \mu_{2, n} R_{2, n}=0, \\
& \lambda_{1, n} \Delta_{n}=0, \quad \lambda_{2, n}\left(1-\Delta_{n}\right)=0
\end{aligned}
$$

for all $j=1,2,3$ and $n=1,2, \ldots, N$, and all Lagrangian multipliers are non-negative due to dual feasibility conditions. The following properties of an optimal policy can be found using the KKT conditions above.

Property 1: At all epochs prior to an empty battery instance for node $T_{k}, k=1,2$, at least one of the constraints in (4d) and (4e) involving $p_{k, n}$ must be met with equality. This property follows from (8c) and the respective complementary slackness conditions.

Property 2: At all epochs prior to empty battery instances for both $T_{1}$ and $T_{2}$, the sum-rate constraint in (4e) must be tight. This property is a consequence of Property 1 and the fact that the constraints in (4d) and (4f) cannot simultaneously be tight without violating (4e).

Property 3: At all epochs prior to an empty battery instance for $T_{3}$, the constraints in (4b), (4c) and (4f) involving $p_{3, n}$ cannot be loose. This property essentially prevents the relay from transmitting with higher rates than necessary, and follows from $(8 \mathrm{e})$.

So far, these properties advocate an energy efficiency perspective. Properties 1 and 3 ensure that if node $T_{1}, T_{2}$ or $T_{3}$ will be energy-deprived in the future, it should not invest energy in improving a rate constraint that is loose, i.e., at least one of the constraints should be tight at the time. Next, we find a property suggesting that at least one of the phases for both messages must be limiting for the intended rate.

Property 4: At all epochs prior to some empty battery instance for $T_{1}, T_{2}$ and $T_{3}$, at least one of the constraints in (4d) and (4f) must be tight for each $k=1,2$. This property is found by combining the stationarity conditions in (8a) and (8b) with Property 2 , and indicates that only a corner point of the multiple access channel capacity region can be used at any time prior to empty batteries for all nodes.

Above properties outline a set of necessary conditions for the optimal policy, but they do not suffice to completely describe the optimal solution. Also note that, unlike previous scheduling work on energy harvesting networks, the nodes do not necessarily have to consume all the harvested energy, since either the transmitters or the relay may be the bottleneck of the system. This may lead to some of the properties not being useful for the majority of the transmission period, since they require some or all batteries to be empty at a future time. Therefore, we find it necessary to explore iterative algorithms to solve the convex sum-rate maximization problem in (4), which will be presented in Section IV.

\section{Subgradient Descent Method}

The convexity of the problem in (4) suggests the use of simple convex optimization algorithms such as gradient descent. However the problems are high-dimensional since the rates for each epoch, i.e., $R_{1, n}, R_{2, n}$, are also optimization parameters along with transmit powers. Moreover, another optimization problem arises when projecting the gradient onto the feasible set at each iteration in order to keep the solution within the feasible set. This optimization problem turns out to be as complicated as the original one, and thus a straightforward gradient descent implementation does not prove to be computationally feasible.

Instead, we rewrite the TWRC sum-rate maximization problem (4) by moving the rate constraints into the objective as

$$
\begin{aligned}
\max _{\substack{\mathbf{p}_{1}, \mathbf{p}_{2}, \mathbf{2} \\
\mathbf{p}_{3}, \mathbf{\Delta}}} & \sum_{n=1}^{N} l_{n} \min \left\{\begin{array}{c}
\Delta_{n} C\left(\frac{h_{13}^{2} p_{1, n}+h_{23}^{2} p_{2, n}}{\Delta_{n}}\right), \\
\Delta_{n} C\left(\frac{h_{13}^{2} p_{1, n}}{\Delta_{n}}\right)+\left(1-\Delta_{n}\right) C\left(\frac{h_{13}^{2} p_{3, n}}{1-\Delta_{n}}\right), \\
\Delta_{n} C\left(\frac{h_{23}^{2} p_{2, n}}{\Delta_{n}}\right)+\left(1-\Delta_{n}\right) C\left(\frac{h_{23}^{2} p_{3, n}}{1-\Delta_{n}}\right), \\
\left(1-\Delta_{n}\right)\left(C\left(\frac{h_{13}^{2} p_{3, n}}{1-\Delta_{n}}\right)+C\left(\frac{h_{23}^{2} p_{3, n}}{1-\Delta_{n}}\right)\right)
\end{array}\right\} \\
\text { s.t. } & \sum_{i=1}^{n} l_{i} p_{j, i} \leq \sum_{i=1}^{n} E_{j, i}, \\
& \sum_{i=1}^{n} E_{j, i}-\sum_{i=1}^{n-1} l_{i} p_{j, i} \leq E_{j, \max }, \quad(10 \mathrm{~b}) \\
& 0 \leq \Delta_{n} \leq 1, \quad j=1,2,3, \quad n=1,2, \ldots, N . \quad(10 \mathrm{~d})
\end{aligned}
$$

The resulting problem has linear constraints and is lower dimensional as the parameter space is reduced to transmit powers and time-sharing parameters only. Note that the objective in (10) remains concave after this mathematical manipulation as the rate for each epoch is the minimum of a set of concave functions. However, the objective in this case, although continuous, is not continuously differentiable, which is necessary for gradient descent. Thus, we instead employ a subgradient descent algorithm which converges to the optimal solution for such problems [16].

The subgradient method is the same as gradient descent, except a subgradient is computed at every iteration instead of the gradient as the gradient may not always exist. If the objective is differentiable at the solution of the current iteration, then the gradient exists and is equal to the subgradient at that point. The algorithm calculates the next solution and projects it onto the feasible set. The optimization problem that arises during this projection is a quadratic program which is easier to solve compared to the projection for the unmodified problem. Lastly, a line search is performed to find the optimal step size in each iteration. Numerical examples are given in Section V where optimal power policies are computed with the subgradient descent method described here.

\section{Numerical Results}

In this section, we present simulations demonstrating the optimal policy for the decode-and-forward two-way relay channel in Section II and a performance comparison with respect to upper and lower bounds. 


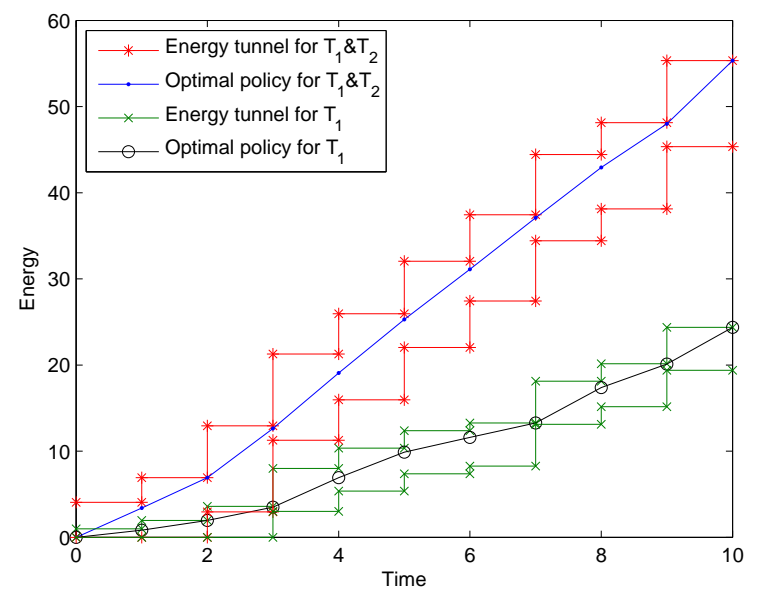

(a)

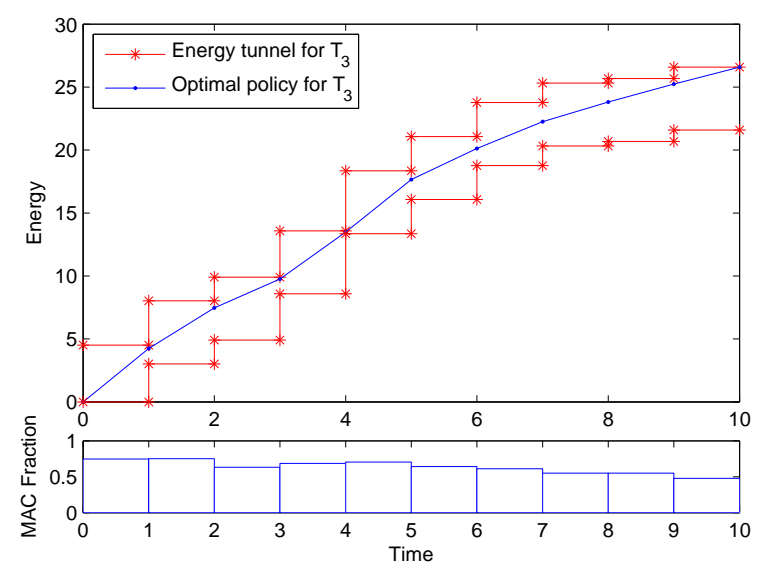

(b)

Fig. 3: Optimal cumulative harvested energy and average consumed energy policies for nodes $T_{1}$ and $T_{1}+T_{2}$ (a) and node $T_{3}$ (b) for a symmetric half-duplex channel with $h_{13}=h_{23}=1$, peak energy harvesting rates $E_{h, 1}=E_{h, 2}=$ $E_{h, 3}=5$ and battery sizes $E_{1, \max }=E_{2, \max }=E_{3, \max }=5$.

Some examples for the optimal power policies found using the subgradient descent algorithm described in Section IV are shown in Figures 3 and 4 for the half-duplex mode with symmetric and asymmetric channel gains. The energy policies, i.e., the total energy consumed since the beginning of transmission, are plotted for $T_{1}$ only and the sum of $T_{1}$ and $T_{2}$ in Figures $3 \mathrm{a}$ and $4 \mathrm{a}$, and for $T_{3}$ in Figures $3 \mathrm{~b}$ and $4 \mathrm{~b}$. The time sharing parameters $\Delta_{i}$ are shown below Figures $3 \mathrm{~b}$ and $4 \mathrm{~b}$. Here, the staircase tunnels represent the energy feasibility constraints for respective nodes, and an energy policy that remains within this tunnel is considered feasible. It is observed that unlike previous work, e.g., for the single user channel in [2], the optimal policy does not necessarily follow the shortest path in the tunnel for all nodes. In Figure 4, it can be

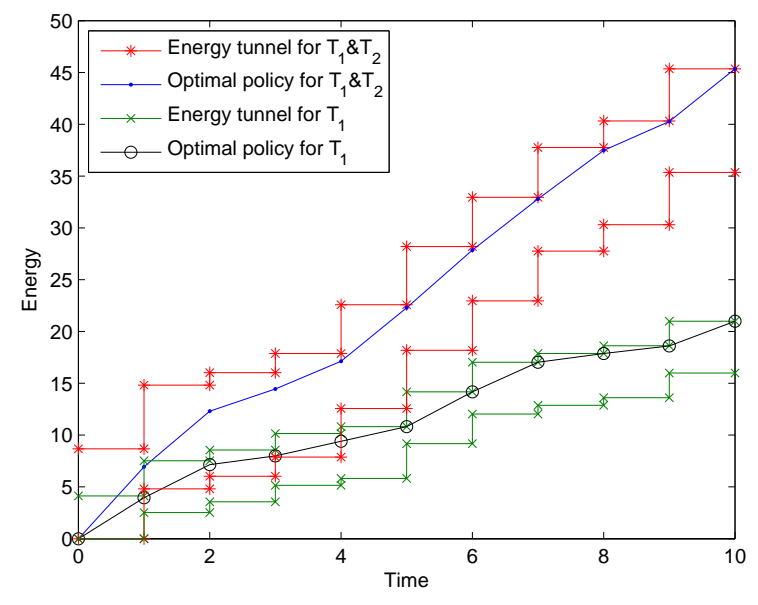

(a)

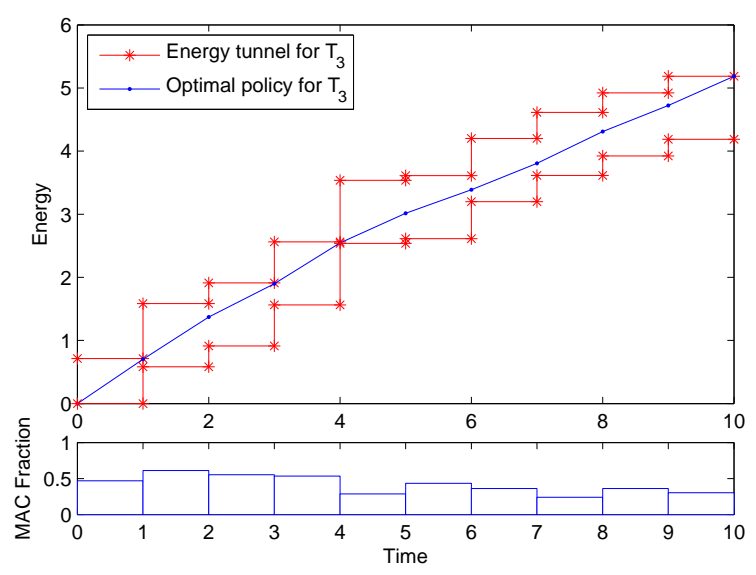

(b)

Fig. 4: Optimal cumulative harvested energy and average consumed energy policies for nodes $T_{1}$ and $T_{1}+T_{2}$ (a) and node $T_{3}$ (b) for an asymmetric half-duplex channel with $h_{13}=1, h_{23}=0.5$, peak energy harvesting rates $E_{h, 1}=5$, $E_{h, 2}=5$ and $E_{h, 3}=1$ and battery sizes $E_{1, \max }=5$, $E_{2, \max }=5$ and $E_{3, \max }=1$.

seen that the second phase of transmission, i.e., the broadcast phase, dominates the other since the relay is energy-deprived in comparison to $T_{1}$ and $T_{2}$. As a result, more time is allocated to the broadcast phase, and the power allocation of $T_{3}$ behaves similar to the shortest path through the tunnel in Figure $4 \mathrm{~b}$ to achieve the optimal broadcast sum-rate. Similarly, the MAC phase emerges as the bottleneck in Figure 3, and the sumpower for $T_{1}$ and $T_{2}$ follows the shortest path through the feasible tunnel.

In Figures 3 and 4, it can also be seen that the time division parameter $\Delta_{n}$ plays an important role in that, by properly selecting $\Delta_{n}$, the effect of unbalanced energy harvests at the transmitters and the relay can be mitigated and the achievable 


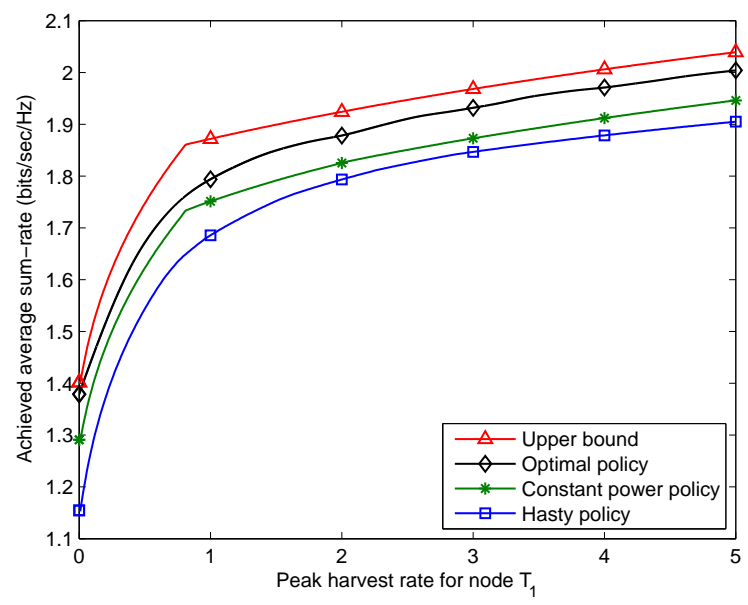

Fig. 5: Average sum-rate achieved against varying peak harvest rates for node $T_{1}$ with $E_{h, 2}=5=E_{h, 3}=5$.

rates in the first and second phases can be balanced. This results in an efficient use of the energy and a higher throughput compared to equal or fixed allocation of phases.

In Figure 5, the average sum-rate achieved by the optimal policy is given for varying peak harvest rates for user $T_{1}$. The peak harvest rates for the other nodes, i.e., node $T_{2}$ and the relay are set to 5 . For comparison, an upper bound and sum-rates achieved by two naïve policies are also plotted. The upper bound corresponds to the case where all the energy harvested throughout transmission is assumed to be received at the beginning of the transmission and the batteries are infinite. This way, the energy causality and battery constraints are removed and the optimal policy is chosen from a larger feasible set. In the hasty policy, the nodes spend their harvested energy within the epoch that follows the energy arrival and never store energy for future epochs. Therefore, a new constraint is introduced, i.e. the batteries must be empty just before an energy arrival, and the feasible set is smaller. The constant power policy attempts to transmit with a fixed average power in each epoch, determined as the average harvesting rate. If the required energy is not available at the node, all remaining energy is consumed in the epoch. The optimal time sharing parameter $\Delta_{i}$ is found in each time slot after the average power values are determined. We remark that the hasty policy is optimal when the nodes do not have a battery, and the constant power policy is asymptotically optimal for large $N$ when the battery capacity is infinite, making the two policies good metrics for comparison. As can be observed in Figure 5, the achieved sum-rate curve approaches the upper bound, while notably outperforming the hasty policy and the constant power policy.

\section{CONCLUSION}

In this paper, we considered a two-way relay channel with energy harvesting and battery limited nodes. We solved the sum-rate maximization problem with a decode-and-forward relay operating in half-duplex mode, and derived insightful properties of the optimal policy through KKT analysis. With some mathematical manipulation, the optimization problem was simplified and solved by an iterative subgradient descent method. Numerical examples were presented to demonstrate the performance of optimal transmission policies. The policies studied in this problem were offline policies. Online policies for the two-way relay based on insights drawn from our results are an interesting future direction, as well as full-duplex operation and relaying strategies.

\section{REFERENCES}

[1] J. Yang and S. Ulukus, "Optimal packet scheduling in an energy harvesting communication system," Communications, IEEE Transactions on, vol. 60, no. 1, pp. 220-230, 2012.

[2] K. Tutuncuoglu and A. Yener, "Optimum transmission policies for battery limited energy harvesting nodes," IEEE Transactions on Wireless Communications, vol. 11, no. 3, pp. 1180-1189, Mar. 2012.

[3] O. Ozel, K. Tutuncuoglu, J. Yang, S. Ulukus, and A. Yener, "Transmission with energy harvesting nodes in fading wireless channels: Optimal policies," IEEE Journal on Selected Areas in Communications, vol. 29 , no. 8, pp. $1732-1743$, Sep. 2011.

[4] K. Tutuncuoglu and A. Yener, "Communicating with energy harvesting transmitters and receivers," in Proceedings of the 2012 Information Theory and Applications Workshop, ITA'12, Feb. 2012.

[5] J. Yang and S. Ulukus, "Optimal packet scheduling in a multiple access channel with energy harvesting transmitters," Communications and Networks, Journal of, vol. 14, no. 2, pp. 140-150, 2012.

[6] J. Yang, O. Ozel, and S. Ulukus, "Broadcasting with an energy harvesting rechargeable transmitter," Wireless Communications, IEEE Transactions on, vol. 11, no. 2, pp. 571-583, 2012.

[7] O. Ozel, J. Yang, and S. Ulukus, "Optimal broadcast scheduling for an energy harvesting rechargeable transmitter with a finite capacity battery," Wireless Communications, IEEE Transactions on, vol. 11, no. 6, pp. 2193-2203, 2012.

[8] M. Antepli, E. Uysal-Biyikoglu, and H. Erkal, "Optimal packet scheduling on an energy harvesting broadcast link," IEEE Journal on Selected Areas in Communications, vol. 29, no. 8, pp. 1721-1731, 2011.

[9] K. Tutuncuoglu and A. Yener, "Sum-rate optimal power policies for energy harvesting transmitters in an interference channel," JCN Special Issue on Energy Harvesting in Wireless Networks, vol. 14, no. 2, pp. 151-161, April 2012.

[10] D. Gunduz and B. Devillers, "Two-hop communication with energy harvesting," in Computational Advances in Multi-Sensor Adaptive Processing (CAMSAP), 2011 4th IEEE International Workshop on. IEEE, 2011, pp. 201-204.

[11] O. Orhan and E. Erkip, "Energy harvesting two-hop networks: Optimal policies for the multi-energy arrival case," in 35th IEEE Sarnoff Symposium (SARNOFF). IEEE, 2012, pp. 1-6.

[12] S. Reddy and C. R. Murthy, "Duty cycling and power management with a network of energy harvesting sensors," in Proceedings of 4th IEEE International Workshop on Computational Advances in Multi-Sensor Adaptive Processing, CAMSAP, San Juan, Puerto Rico, Dec. 2011.

[13] Z. Mao, C. Koksal, and N. Shroff, "Near optimal power and rate control of multi-hop sensor networks with energy replenishment: Basic limitations with finite energy and data storage," IEEE Transactions on Automatic Control, vol. 57, no. 4, pp. 815-829, 2012.

[14] D. Gunduz, E. Tuncel, and J. Nayak, "Rate regions for the separated two-way relay channel," in Communication, Control, and Computing, 2008 46th Annual Allerton Conference on. IEEE, 2008, pp. 13331340.

[15] S. Kim, N. Devroye, P. Mitran, and V. Tarokh, "Achievable rate regions and performance comparison of half duplex bi-directional relaying protocols," Information Theory, IEEE Transactions on, vol. 57, no. 10, pp. 6405-6418, 2011.

[16] D. Bertsekas, Nonlinear programming. Athena Scientific, 1999.

[17] B. Rankov and A. Wittneben, "Achievable rate regions for the twoway relay channel," in Information Theory, 2006 IEEE International Symposium on. IEEE, 2006, pp. 1668-1672. 